\title{
The effect of implementing pre-surgical ultrasound-guided fine- needle aspiration biopsy on thyroid surgery, a 6-year interrupted time series analysis in Qilu Hospital of Shandong University
}

\author{
Zhiyan Liu ${ }^{1,2 \#}$, Shaofeng Sui ${ }^{3 \#}$, Peng $\mathrm{Su}^{2}$, Xiaofang Zhang ${ }^{2}, \mathrm{Jing} \mathrm{Hu}^{2}$, Feifei Sun ${ }^{2}$, Bo Han ${ }^{2}$ \\ ${ }^{1}$ Department of Pathology, Shanghai Jiao Tong University Affiliated Sixth People's Hospital, Shanghai, China; ${ }^{2}$ Department of Pathology, Qilu \\ Hospital of Shandong University, Shandong University, Jinan, China; ${ }^{3}$ Department of Occupational and Environmental Health Monitoring and \\ Assessment, Shandong Center for Disease Control and Prevention, Jinan, China \\ Contributions: (I) Conception and design: Z Liu, S Sui; (II) Administrative support: B Han; (III) Provision of study materials or patients: P Su; (IV) \\ Collection and assembly of data: P Su; (V) Data analysis and interpretation: S Sui; (VI) Manuscript writing: All authors; (VII) Final approval of \\ manuscript: All authors. \\ \#These authors contributed equally to the work. \\ Correspondence to: Zhiyan Liu, MD, PhD. Department of Pathology, Shanghai Jiao Tong University Affiliated Sixth People's Hospital, Shanghai, \\ China. Email: zhiyanliu@hotmail.com.
}

Background: Thyroid fine-needle aspiration biopsy (FNAB) is not well developed in most of the large academic hospitals in China, including Qilu Hospital of Shandong University. Ultrasound-guided FNAB (UG-FNAB) was initiated as a pre-surgical diagnostic method in 2015 in Qilu Hospital, and the current study evaluates its impact on the number of thyroid surgeries and the ratio of malignancy (ROM) in surgically resected cases, comparing the post and pre-UG-FNAB periods.

Methods: Thyroid surgery and UG-FNAB data for the study period (Jan 2013 to Dec 2018) were collected from the hospital information system (HIS). A natural experimental approach using segmented linear regression was performed on the interrupted time-series (ITS) data to estimate the level-change of the number and the trend-change of ROM of surgically resected cases following the implementation of UGFNAB diagnosis.

Results: The number of UG-FNAB cases in Qilu Hospital increased rapidly from 1,367 in 2015 to 3,402 in 2018, with an average annual increase of 678 cases. Comparing the pre-UG-FNAB and post-UGFNAB periods, the overall ROM of thyroid resections increased from $58.7 \%$ to $73.2 \%(\mathrm{P}<0.01)$. Due to the implementation of UG-FNAB, the regression line of surgical cases showed an inflection point in March 2015, and the slope of the regression line of post-intervention was lower than that of pre-intervention. For each additional month, the ROM in thyroid surgical cases increased by $0.389 \%(\mathrm{P}<0.01)$. After excluding the influence of time, the ROM in surgically resected thyroid cases increased by $4.781 \%$ due to the application of the UG-FNAB examination $(\mathrm{P}<0.01)$.

Conclusions: This successful implementation of UG-FNAB further improved overall ROMs in surgically resected cases particularly as we acquired further experience with the procedure and reduced unnecessary diagnostic surgeries for patients with benign and borderline lesions.

Keywords: Thyroid; diagnostic surgery; FNA cytology; risk of malignancy; interrupted time series analysis

Submitted Mar 13, 2020. Accepted for publication Jun 18, 2020.

doi: $10.21037 /$ gs-20-348

View this article at: http://dx.doi.org/10.21037/gs-20-348

^ Zhiyan Liu, ORCID: 0000-0001-9668-7756; Shaofeng Sui, ORCID: 0000-0002-8476-2410. 


\section{Introduction}

The incidence of detecting thyroid nodules is increasing globally, with a detection rate of $20-76 \%$ in the population. Most nodules are benign and have no adverse health effects, while $7-15 \%$ of thyroid nodules are malignant (1-3). The focus of the clinical workup is to identify thyroid carcinomas and accurately distinguish them from nonmalignant thyroid nodules, strike a balance between surgery and clinical follow-up, and reduce non-essential surgical treatment. UG-FNAB is a traditional minimally invasive diagnostic technique, which can identify the nature of thyroid nodules preoperatively and provide the basis for individualized and accurate treatment of thyroid disease (4). It is currently considered the most reliable method to predict benign and malignant thyroid nodules preoperatively and consequently avoid unnecessary diagnostic thyroid surgeries. UG-FNAB is recommended as the pre-surgical diagnostic method of choice according to the guidelines developed by the American Thyroid Association (ATA), American Academy of Clinical Endocrinologists, and many others (5-8). Although UG-FNAB has the above advantages, its clinical value is still controversial, and its impact on the ROM of surgical cases remains unclear. According to the literature we reviewed, it has not been reported whether the UG-FNAB diagnosis can reduce the number of surgical cases and improve the ROM of surgical thyroid lesions.

The purpose of this study is to evaluate the impact of UG-FNAB on the number of thyroid surgeries and the proportion of malignant tumors in surgical cases and to verify whether UG-FNAB examination achieved the vision of "Only suspicious nodule should undergo diagnostic surgery (Benign disease should be handled conservatively)," which is the equivalent of "First do not harm patients (Primum non nocere by the Hippocratic oath)" in Western practice.

\section{Methods}

\section{Study population and data source}

We retrospectively collected data on patients who underwent thyroid surgery and UG-FNAB from the hospital information system (HIS) of Qilu Hospital of Shandong University for the period Jan 2013 to Dec 2018. No human investigations or animal experiments were included in this study, so ethical approval was not required. Qilu Hospital is the largest general hospital in Jinan city, with 3,800 beds, 3.8 million outpatient visits, and 97,000 surgeries in 2018. The HIS data contains admission records which include the information of the patients, such as age, gender, inpatient area, admission number, the pathological diagnosis of thyroid lesion, date of surgery, etc. A total of 10,495 patients were enrolled in this study and included 3,317 from the pre-UG-FNAB period and 7,178 from the post UG-FNAB period.

\section{Fine-needle aspiration biopsy diagnoses}

FNAB is the most accurate and cost-effective method for evaluating thyroid nodules. Sonographic examination was available for all the patients in or outside Qilu Hospital. The recommendation of diagnostic thyroid FNA of a thyroid nodule based on the sonographic pattern suggested in the 2015 ATA management guidelines for adult patients with thyroid nodules and differentiated thyroid cancer and the Chinese consensus and management guideline of ultrasound guided thyroid fine needle aspiration $(7,9)$. An experienced technician of FNA operator together with one of ten endocrinologists who was on duty for sonographic examination performed UG-FNAB. After applying a coupling agent, the ultrasonic probe is wrapped with disposable protective film and sterilized. Patients lie down in a supine position with the neck slightly extended. After nodules localization, the neck is sterilized and draped. Under ultrasound guidance, the needle is introduced into the thyroid nodules. The biopsy is performed with to-andfro movements in the nodule several times to complete the sampling. Aspirated material is placed on the glass slides, smeared, fixed in ethanol and stained with hematoxylineosin. Repeat the procedure as needed by gross evaluation of sample. Usually, 2 to 3 passes are employed per nodule. After the sampling, the patient is placed under medical observation for 10 to 20 minutes in the observation area (6).

Cytological samples were diagnosed by three pathologists (ZL, PS, and XZ) according to the new Bethesda Reporting System for Thyroid Cytopathology, and no rapid on-site evaluation was performed because of shortage of pathologist $(10,11)$. Cytological consultation was recommended for those patients who underwent thyroid FNA in outside hospitals, and repeat $\mathrm{FNAB}$ was rendered for those diagnostic categories recommended by 2015 ATA management guidelines for thyroid nodules (7). $B R A F^{V 600 E}$ genetic testing is not carried out routinely on cytological specimens in Qilu Hospital since these expensive molecular tests are not covered by the health insurance system and the expense is covered directly by the patients in China $(7,12,13)$. 
Table 1 Baseline characteristics of thyroid surgery patients enrolled in this study

\begin{tabular}{|c|c|c|c|c|}
\hline Characteristic & Pre-UG-FNAB & Post-UG-FNAB & Total & $P$ value \\
\hline Patients, n (\%) & 3,367 (31.9) & $7,178(68.1)$ & $10,545(100.0)$ & - \\
\hline Age (years), (mean $\pm S D$ ) & $47.3 \pm 12.5$ & $47.0 \pm 12.5$ & $47.1 \pm 12.5$ & $0.403^{\triangle}$ \\
\hline \multicolumn{5}{|l|}{ Sex, n (\%) } \\
\hline Male & $823(24.4)$ & $1,811(25.2)$ & $2,634(25.0)$ & \\
\hline \multicolumn{5}{|l|}{ Thyroid surgery, n (\%) } \\
\hline Malignancy & $1,976(58.7)$ & $5,256(73.2)$ & $7,232(68.6)$ & $0.000^{\#}$ \\
\hline Benign & $1,391(41.3)$ & 1,922 (26.8) & 3,313 (31.4) & \\
\hline
\end{tabular}

${ }^{\triangle}$, one-way ANOVA analysis; ", Pearson Chi-Square test.

\section{Statistical analysis}

A segmented linear regression was performed on the interrupted time-series (ITS) data to estimate two parameters of interest associated with the UG-FNAB implementation: (I) level-change in the subsequent outcome and (II) trend-change in subsequent (14). The pre-intervention period was defined from January 2013 to February 2015 (26 months). The post-intervention period included monthly time points from March 2018 to December 2018 (46 months). The regression model was specified as follow:

$\mathrm{Y}=\beta 0+\beta 1 \times$ time $+\beta 2 \times \mathrm{UG}-\mathrm{FNAB}+\beta 3 \times \mathrm{UG}-\mathrm{FNAB}$ $x$ time

$\beta 0$ estimates the baseline level of the outcome just before the beginning of the time series. $\beta 1$ estimates the pre-UGFNAB intervention trend, $\beta 2$ estimates the level-change between the time point immediately before $v$ s. after the UG-FNAB intervention, and $\beta 3$ estimates the trend-change occurring immediately after the UG-FNAB intervention.

Analyses were based on 26 pre-intervention data points (Jan. 2013-Feb. 2015) and 46 post-intervention data points (Mar. 2015-Dec. 2018). The final model specification was derived using the Akaike information criterion (AIC) to judge the merits and disadvantages of the model and select the regression terms that went into the equation in order to maximize statistical power. The level-change model was more applicable to analyze the rates of malignant surgical cases of thyroid in Qilu hospital. By removing the interaction term of $\mathrm{UG}-\mathrm{FNAB} \times$ time out of the regression equation, the AIC value became smaller. Durbin-Watson statistics indicated no significant autocorrelation in the model $(\mathrm{P}>0.05)$. All analyses were carried out using $\mathrm{R}$ 3.5.1 software (R Development Core Team, Austria).

\section{Results}

Of the 10,545 surgically treated patients with thyroid nodules enrolled in this study, 3,367 constituted the preUG-FNAB group and 7,178 constituted in the post-UGFNAB group. Of total patients, 7,911 were female (75.0\%) and 2,634 were male (25.0\%). The mean age was 47.1 years (range, 15-84 years). The difference in average age between the two groups was not significant $(\mathrm{P}=0.403)$, as well as the male/female ratio $(\mathrm{P}=0.384)$. We observed an increase of $14.5 \%$ in the overall ROM histologically between pre- and post-UG-FNAB groups. Pearson Chi-square test indicates a significant difference comparing the ROMs of the preand post-implementation groups (ROM: $58.7 \%$ vs. $73.2 \%$; $\mathrm{P}=0.000)$. The baseline characteristics of patients enrolled in this study was shown in Table 1.

In order to minimize missing malignancy, the clinical management of the thyroid nodule will be made by the physician combining cytological diagnosis, sonographic feature and clinical risk factors. If the first cytological examination could not clarify the nature of the lesion, the repeat UG-FNAB puncture was required, especially for non-diagnostic, benign and indeterminate diagnostic category. If the cytology is reported as malignancy, surgery will be recommended for the patient except for malignant lymphoma and metastatic malignancies. If the cytology is reported as suspicious for malignancy, surgical management should be similar to that of malignant cytology, depending 


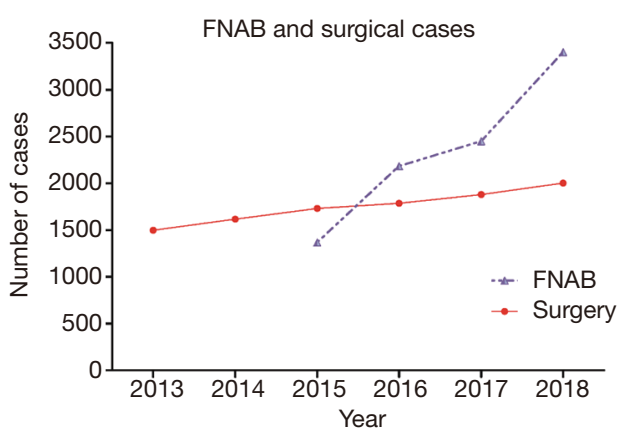

Figure $1 \mathrm{UG}-\mathrm{FNAB}$ and thyroid surgical cases in Qilu Hospital since 2013 to 2018. UG-FNAB, ultrasound-guided fine-needle aspiration biopsy.

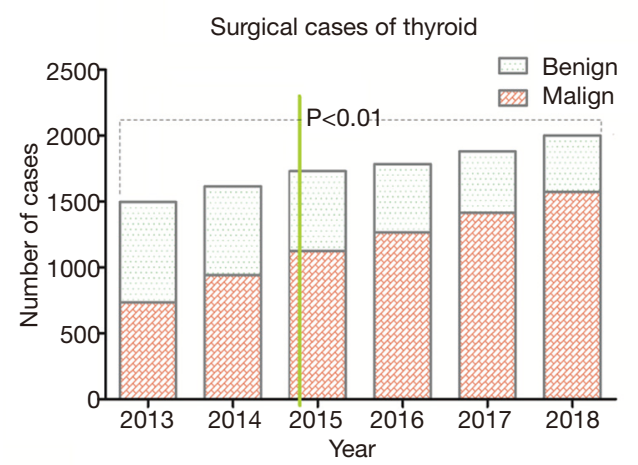

Figure 2 Benign and malignant surgical cases of thyroid in Qilu Hospital during the pre- and post-intervention period since 2013 to 2018. March of 2015 was the first month of UG-FNAB taken place in Qilu Hospital. UG-FNAB, ultrasound-guided fine-needle aspiration biopsy.

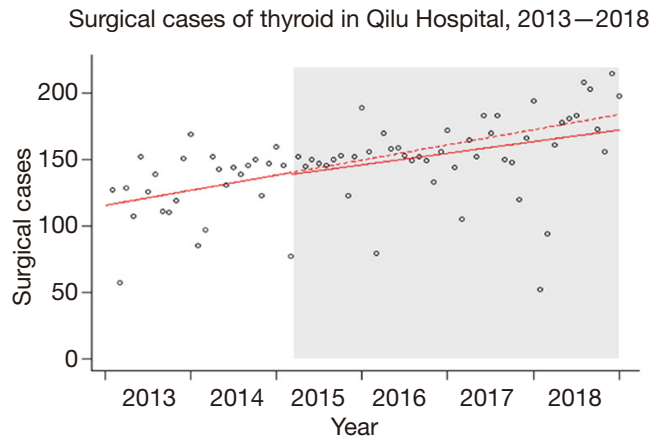

Figure 3 Surgical cases in Qilu Hospital per month since 2013 to 2018. The gray color area is the post-UG-FNAB intervention period. The dashed curve means the anticipated number of cases by prolonged the regression line before UG-FNAB operation. The solid line means the anticipated value of actual number of cases. UG-FNAB, ultrasound-guided fine-needle aspiration biopsy. on clinical risk factors, sonographic features, and patient preference $(7,9)$. By far, there was no missing malignancy nor malpractice litigation during the study period.

The number of surgical cases increased from 1,497 in 2013 to 2,002 in 2018, an average increase of 101 cases per year in Qilu Hospital (Figure 1). The curve of surgical cases was relatively flat or slightly sloping. The number of UGFNAB cases in Qilu Hospital increased rapidly from 1,367 in 2015 to 3,402 in 2018, with an average annual increase of 678 cases. The curve of UG-FNAB cases was relatively steep.

Meanwhile, the proportion of malignant tumors in surgical cases increased year by year from 2013 to 2018 (Figure 2), and the proportion of malignancy increased gradually. It can be seen that in 2013 , before the intervention, more than half $(50.9 \%)$ of surgically treated patients had benign tumors that would not have required surgical treatment and underwent thyroid lobectomy only for diagnostic purposes. In 2018, after the intervention, only $21.3 \%$ of patients underwent surgery for benign lesions, especially non-neoplastic lesions, which suggested that significant numbers of patients with benign lesions were spared diagnostic surgeries. The pale green vertical line in Figure 2, which divided all surgical cases into preintervention and post-intervention, represented the beginning of the UG-FNAB examination-March 2015. The ROM in all surgically treated thyroid nodules between the pre- and post-intervention periods were statistically significantly different $(58.7 \%$ vs. $73.2 \%)(\mathrm{P}<0.01)$. Thus, more efficient triage of patients with malignant tumors for surgery was achieved particularly in later years as we acquired more experience.

Due to the implementation of UG-FNAB, the regression line of surgery cases showed an inflection point in March 2015, and the slope of the regression line of post-intervention was lower than that of pre-intervention (Figure 3). This indicates that the predicted value based on the actual number of surgical cases after the intervention (the solid line in the gray area) is somewhat lower than the predicted value based on the monitoring data before the implementation of FNAB (the dotted line in the gray area). In other words, the implementation of UG-FNAB has, to some extent, slowed down the rapid growth of thyroid surgery cases in Qilu Hospital.

As shown in Figure 4, the proportion of malignant tumors in surgical cases increased month by month before and after the implementation of UG-FNAB. ITS Analysis showed significant level change for the ROM of 


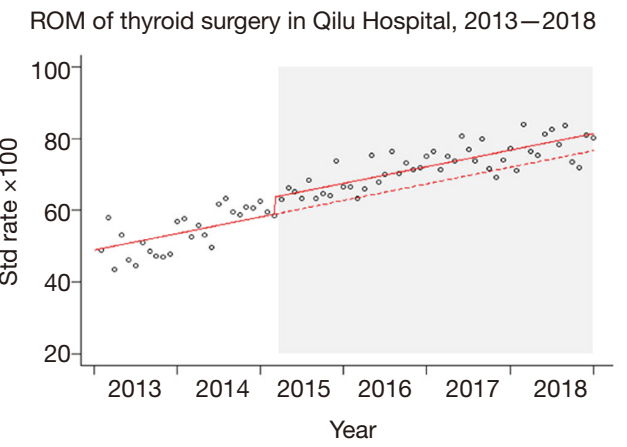

Figure 4 Level-change model of ROMs in Qilu Hospital per month since 2013 to 2018 . The gray color area is the UG-FNAB intervention period. The dashed curve means the anticipated rates by prolonged the regression line before UG-FNAB operation. The solid line means the anticipated values of actual rates. ROM, ratio of malignancy; UG-FNAB, ultrasound-guided fine-needle aspiration biopsy.

Table 2 Results of level-change model of ITS

\begin{tabular}{lcccc}
\hline Items & Estimate & $\mathrm{SD}$ & $\mathrm{Z}$ & $\mathrm{P}$ \\
\hline Intercept & 48.77 & 0.97 & 50.49 & 0.000 \\
UG-FNAB & 4.78 & 1.80 & 2.66 & 0.008 \\
Time (month) & 0.39 & 0.04 & 9.32 & 0.000 \\
\hline
\end{tabular}

ITS, interrupted time-series; UG-FNAB, ultrasound-guided fineneedle aspiration biopsy.

surgical cases $(\mathrm{P}<0.01)$ in March of 2015 (Table 2). The dotted line shown in the gray area of Figure 4 represented the regression line of a possible ROM if UG-FNAB was not implemented. The solid line represents the ROM regression line that occurred after the implementation of UG-FNAB. It can be seen that UG-FNAB intervention significantly increased the proportion of malignancy cases in surgical cases. By the end of 2018, the proportion of malignant tumors had increased to about $80 \%$ of thyroid surgeries.

ITS Analysis showed that UG-FNAB examination and time factors had statistically significant impacts on the increase of malignancy proportion in surgical cases $(\mathrm{P}<0.01)$. After excluding the influence of time, the proportion of malignant tumors in surgical cases increased by $4.781 \%$ due to the application of UG-FNAB technique. For each additional month, the proportion of malignant tumors in surgical cases increased by $0.389 \%(\mathrm{P}<0.01)$.

\section{Discussion}

Thyroid nodules are classified into benign, borderline (tumors of uncertain malignant potential and noninvasive follicular neoplasm with papillary-like nuclear features) and malignant tumor categories according to their morphological features (presence or absence of PTC type nuclear features, invasion, cellular atypia, necrosis, and inflammatory background), in the 2017 WHO classification of thyroid tumor (15-17). Most patients with benign lesions need only regular clinical follow-up, and no surgical treatment is required, especially those with non-neoplastic benign lesions. The progression of the malignancy will not only lead to poor quality of life but also endanger patients' lives, especially in high-risk thyroid carcinoma $(11,18,19)$. Early detection of thyroid carcinoma is particularly important because of its high incidence of lymph node metastasis and extra-thyroid extension risks $(11,18,19)$. Surgical excision and pathological examination are the gold standard for the diagnosis of thyroid nodules, and the operation modes are different for benign tumors, borderline tumors, low-risk and high-risk carcinomas according to the ATA guideline $(7,20,21)$. Therefore, preoperative diagnosis is very important to properly triage the patients and select appropriate treatment. UG-FNAB is one of the first line diagnostic methods widely used in the diagnosis of the thyroid lesions, which helps in determining the need for surgery and the operation methods (22-24). A large number of studies have reported that the sensitivity of UG-FNAB for the diagnosis of thyroid nodules is $73.5-100 \%$, the specificity is $50.7-97.0 \%$, the accuracy is $68.8-98.8 \%$, the positive predictive value is $55.9-98.7 \%$, and the negative predictive value $96.3-100.0 \%(7,25,26)$.

As shown in Figure 2 and Table 1, ROM is high in both post- and pre-UG-FNAB periods in Qilu Hospital. This is probably due to the differences in clinical management of thyroid nodules in China and the other countries. Vuong et al. recently reported a significant difference in ROM of both cytological and surgical practice geographically, which ranged from $14.8-55.7 \%$ in surgical practice in Western Countries and $15.0-91.8 \%$ in Asian countries (27). This is probably due to the different management criteria of thyroid lesions in different countries (20,21,28-30). Such as, thyroid UG-FNAB has not been well developed in most of the large academic hospitals in China, although both UG-FNAB and frozen section were recommended as diagnostic methods for thyroid nodules by Chinese Medical 
Association management guideline (CMAMG) $(28,29)$. This leads to the wide use of frozen section in thyroid nodule work up in China, and this was also the practice in Qilu Hospital before 2015 (11).

Nodules with malignant and suspicion sonographic pattern will directly undergo diagnostic surgery and frozen sections to confirm the diagnosis during surgery in pre-UG-FNAB period in Qilu Hospital. However, in post-UG-FNAB period, those nodules with high suspicion sonographic pattern will further undergo UGFNAB to confirm malignancy. As a result, the frequency of intraoperative frozen section diagnosis decreased dramatically, and is now mainly used to confirm lymph node metastasis. In China, the management of PTC measuring $\leq 10 \mathrm{~mm}$ will depend on the patient's decision based on a discussion between the patient and his treating physician, which is similar to the active surveillance as recommended by the ATA guidelines and the Japanese Association of Endocrine Surgery clinical guidelines (7,31).

The number of UG-FNAB and thyroid surgery has obviously increased since UG-FNAB was implemented $\mathrm{g}$ as the pre-surgery diagnostic procedure in March 2015 (Figure 1). The number of thyroid surgeries steadily increased from 1,479 in 2013 to 2,002 in 2018. Those patients with non-neoplastic lesions and without clinical symptoms will no longer undergo surgery. For example, there were totally 3,402 cases of thyroid UG-FNAB and only 2,002 cases underwent thyroid surgery in 2018. Those diagnosed as malignant by UG-FNAB will undergo surgery and constituted a large number of thyroid surgeries, which resulted in the dramatic overall increase of ROMs from $58.7 \%$ to $73.2 \%$ (Figure 2). These results further confirmed that UG-FNAB is suitable for large-scale screening of thyroid nodules and can reduce unnecessary diagnostic surgery.

Comparing post- and pre-UG-FNAB periods, the overall ROM of thyroid surgery increased by $14.5 \%$. This is an appreciated effect related to the implementation of pre-surgery UG-FNAB in Qilu hospital. ITS analysis provides an effective statistical method that can deduct the contribution of physicians' accumulated experiences over time and separate the influence of UG-FNAB on the ROM of surgical cases. After adjusting for time effect, the ROM of thyroid surgery improved by $4.78 \%$ due to the use of UG-FNAB diagnostic technique. In other words, with the implementation of UG-FNAB, only patients with highly suspected malignant lesions were treated with surgery. This is beneficial to avoid the surgery related morbidity and to reduce the financial burden of patients and the health care system.

Some scholars believe that the accurate UG-FNAB would require a high degree of working experience and technical level of the operator. The higher the technical level of the operator, the better the cellular smears and resulting diagnosis would be $(19,20)$. As the FNAB operators acquired more experience, their skills improved and, the quality of the FNAB cellular smears was also improved in Qilu Hospital. There was only $1.8 \%$ of inadequate samples in 2018 , comparing with $3.0 \%$ in 2015 . The proportion of benign category decreased from $48.0 \%$ in 2015 to $21.0 \%$ in 2018 , and there was no repeat FNAB of this category due to inconsistent sonographic features in 2018. There was also a gradually increasement of the diagnosis of follicular tumor/ suspicious of follicular tumor category. These results further confirmed the improvement of the skill of the operators. These multiple factors contributed to a highly accurate and satisfactory FNAB diagnosis in our practice. The ITS analysis showed that the ROM increased by $0.39 \%$ for each month gradually.

However, our study still has some limitations. First, before 2013, the new outpatient and the Ward building of Qilu Hospital have not been opened yet, and the number of inpatients and surgical cases was small. As a result, the data before and after 2013 were not comparable. Therefore, thyroid surgery data before 2013 were not included in this study, which brought some uncertainty to the conclusions of this study.

\section{Conclusions}

In conclusion, the present study is an example for a successful implementation of UG-FNAB before thyroid surgery. This intervention might serve as a good model for other hospitals in countries where UG-FNAB is not available yet. UGFNAB examination can significantly reduce the number of unnecessary diagnostic surgeries for patients with benign lesions and significantly increase the proportion of malignant tumors in surgical cases. Our experience demonstrated that the overall ROMs in surgical cases improved further with experience, which resulted in minimizing unnecessary diagnostic surgery for patients with benign lesions to less than one quarter of surgically treated patients.

\section{Acknowledgments}

Funding: This work has been supported by National Nature 
Science Foundation of China (Grant No. 81972500) and Natural Science Foundation of Shandong Province, China (Grant No. ZR2019MH024).

\section{Footnote}

Provenance and Peer Review: This article was commissioned by the Guest Editor (Kennichi Kakudo) for the series "Asian and Western Practice in Thyroid Pathology: Similarities and Differences" published in Gland Surgery. The article was sent for external peer review organized by the Guest Editor and the editorial office.

Conflicts of Interest: All authors have completed the ICMJE uniform disclosure form (available at http:// dx.doi.org/10.21037/gs-20-348). The series "Asian and Western Practice in Thyroid Pathology: Similarities and Differences" was commissioned by the editorial office without any funding or sponsorship. The authors have no other conflicts of interest to declare.

Ethical Statement: The authors are accountable for all aspects of the work in ensuring that questions related to the accuracy or integrity of any part of the work are appropriately investigated and resolved. No human investigations or animal experiments were included in this study, so ethical approval was not required.

Open Access Statement: This is an Open Access article distributed in accordance with the Creative Commons Attribution-NonCommercial-NoDerivs 4.0 International License (CC BY-NC-ND 4.0), which permits the noncommercial replication and distribution of the article with the strict proviso that no changes or edits are made and the original work is properly cited (including links to both the formal publication through the relevant DOI and the license). See: https://creativecommons.org/licenses/by-nc-nd/4.0/.

\section{References}

1. Gharib H, Papini E, Paschke R, et al. American Association of Clinical Endocrinologists, Associazione Medici Endocrinologi, and European Thyroid Association medical guidelines for clinical practice for the diagnosis and management of thyroid nodules: Executive Summary of recommendations. Endocrine Practice 2010;16:468-75.

2. Burman KD, Wartofsky L. Clinical practice. Thyroid
Nodules. New Engl J Med 2015;373:2347.

3. Mandel SJ. A 64-year-old woman with a thyroid nodule. JAMA 2004;292:2632-42.

4. Goldfarb M, Gondek S, Solorzano C, et al. Surgeonperformed ultrasound can predict benignity in thyroid nodules. Surgery 2011;150:436-41.

5. Leenhardt L, Erdogan MF, Hegedus L, et al. 2013 European thyroid association guidelines for cervical ultrasound scan and ultrasound-guided techniques in the postoperative management of patients with thyroid cancer. Eur Thyroid J 2013;2:147-59.

6. Tian W, Sun H, He Q. Expert consensus and operating guidelines for ultrasound-guided fine needle biopsy of thyroid nodules (2018 Version) (in Chinese). Chinese Journal of Practical Surgery 2018;38:6-9.

7. Haugen BR, Alexander EK, Bible KC, et al. 2015 American Thyroid Association Management Guidelines for Adult Patients with Thyroid Nodules and Differentiated Thyroid Cancer: The American Thyroid Association Guidelines Task Force on Thyroid Nodules and Differentiated Thyroid Cancer. Thyroid 2016;26:1-133.

8. Gharib H, Papini E, Garber JR, et al. American Association of Clinical Endocrinologists, American College of Endocrinology, and Associazione Medici Endocrinologi Medical Guidelines for Clinical Practice for the Diagnosis and Management of Thyroid Nodules--2016 Update. Endocr Pract 2016;22:622-39.

9. Tian W, Sun H, He Q. The Chinese consensus and management guideline of ultrasound guided thyroid fine needle aspiration. Chin J Pract Surg 2018;38:241-3.

10. Cibas ES, Ali SZ. The 2017 Bethesda System for Reporting Thyroid Cytopathology. Thyroid 2017;27:1341-6.

11. Liu Z, Liu D, Ma B, et al. History and Practice of Thyroid Fine-Needle Aspiration in China, Based on Retrospective Study of the Practice in Shandong University Qilu Hospital. J Pathol Transl Med 2017;51:528-32.

12. Kennichi K, Liu ZY, Hirokawa M. Thyroid FNA Cytology: Differential diagnoses and Pitfills. Japan: Book Way GLOBE, 2017.

13. Rusinek D, Swierniak M, Chmielik E, et al. BRAFV600EAssociated Gene Expression Profile: Early Changes in the Transcriptome, Based on a Transgenic Mouse Model of Papillary Thyroid Carcinoma. PLoS One 2015;10:e0143688.

14. Bernal JL, Cummins S, Gasparrini A. Interrupted time series regression for the evaluation of public health interventions: a tutorial. Int J Epidemiol 2017;46:348-55.

15. Lloyd RV, Osamura RY, Klöppel G, et al. WHO 
classification of tumours: Pathology and genetics of tumours of endocrine organs. 4th ed. Lyon: IARC, 2017.

16. Kakudo K, Bychkov A, Bai Y, et al. The new 4th edition World Health Organization classification for thyroid tumors, Asian perspectives. Pathol Int 2018;68:641-64.

17. Liu Z, Zhou G, Nakamura M, et al. Encapsulated follicular thyroid tumor with equivocal nuclear changes, so-called well-differentiated tumor of uncertain malignant potential: a morphological, immunohistochemical, and molecular appraisal. Cancer Sci 2011;102:288-94.

18. Bai Y, Kakudo K, Li Y, et al. Subclassification of non-solidtype papillary thyroid carcinoma identification of high-risk group in common type. Cancer Sci 2008;99:1908-15.

19. Liu Z, Kakudo K, Bai Y, et al. Loss of cellular polarity/ cohesiveness in the invasive front of papillary thyroid carcinoma, a novel predictor for lymph node metastasis; possible morphological indicator of epithelial mesenchymal transition. J Clin Pathol 2011;64:325-9.

20. Kakudo K, Kameyama K, Miyauchi A, et al. Introducing the reporting system for thyroid fine-needle aspiration cytology according to the new guidelines of the Japan Thyroid Association. Endocr J 2014;61:539-52.

21. Kocjan G, Cochand-Priollet B, de Agustin PP, et al. Diagnostic terminology for reporting thyroid fine needle aspiration cytology: European Federation of Cytology Societies thyroid working party symposium, Lisbon 2009. Cytopathology 2010;21:86-92.

22. Gao L, Ma B, Zhou L, et al. The impact of presence of Hashimoto's thyroiditis on diagnostic accuracy of ultrasound-guided fine-needle aspiration biopsy in subcentimeter thyroid nodules: A retrospective study from FUSCC. Cancer Med 2017;6:1014-22.

23. Lee MJ, Hong SW, Chung WY, et al. Cytological results of ultrasound-guided fine-needle aspiration cytology for thyroid nodules: emphasis on correlation with sonographic

Cite this article as: Liu Z, Sui S, Su P, Zhang X, Hu J, Sun F, Han B. The effect of implementing pre-surgical ultrasoundguided fine-needle aspiration biopsy on thyroid surgery, a 6-year interrupted time series analysis in Qilu Hospital of Shandong University. Gland Surg 2020;9(5):1716-1723. doi: 10.21037/gs-20348 findings. Yonsei Med J 2011;52:838-44.

24. Chung J, Youk JH, Kim JA, et al. Initially non-diagnostic ultrasound-guided fine needle aspiration cytology of thyroid nodules: value and management. Acta Radiol 2012;53:168-73.

25. Xing $M$, Haugen BR, Schlumberger M. Progress in molecular-based management of differentiated thyroid cancer. Lancet 2013;381:1058-69.

26. Labourier E, Shifrin A, Busseniers AE, et al. Molecular Testing for miRNA, mRNA, and DNA on Fine-Needle Aspiration Improves the Preoperative Diagnosis of Thyroid Nodules With Indeterminate Cytology. J Clin Endocrinol Metab 2015;100:2743-50.

27. Vuong HG, Ngo HTT, Bychkov A, et al. Differences in surgical resection rate and risk of malignancy in thyroid cytopathology practice between Western and Asian countries: A systematic review and meta-analysis. Cancer Cytopathol 2020;128:238-49.

28. Association CM. Chinese Medical Association Management Guidelines for Endocrine and metabolic diseases. 2nd ed. Beijing, China: People Hygiene Press, 2009.

29. Association CM. Chinese Medical Association management guidelines for Surgery. 2nd ed. Beijing, China: People Hygine Press, 2011.

30. Neagoe RM, Sala DT, Pascanu I, et al. A comparative analysis of the initial east European center experience with a western high-volume center for open minimally invasive parathyroidectomy (OMIP) as treatment of primary hyperparathyroidism. Acta Endocrinol (Buchar) 2016;12:297-303.

31. Takami H, Ito Y, Okamoto T, et al. Therapeutic strategy for differentiated thyroid carcinoma in Japan based on a newly established guideline managed by Japanese Society of Thyroid Surgeons and Japanese Association of Endocrine Surgeons. World J Surg 2011;35:111-21. 